\title{
HPLC Determination of Quercetin in Three Plant Drugs from Genus Sedum and Conjecture of the Best Harvest Time
}

\author{
Ma Yue-ling ${ }^{1}$, Chen Yu-jie ${ }^{2}$, Wan Ding-rong ${ }^{3}$, Chen ping ${ }^{1}$, and Xu Ran ${ }^{1 *}$
}

\section{Ma Yue-ling ${ }^{1}$, Chen Yu-jie ${ }^{2}$, Wan Ding-rong ${ }^{3}$, Chen ping ${ }^{1}$, and Xu Ran ${ }^{1 *}$ \\ ${ }^{1} S c h o o l$ of biological and pharmaceutical engineering, Wuhan Polytechnic University, Wuhan, 430023, CHINA. \\ ${ }^{2}$ Shandong Center for Drug Evaluation \& Certification, Jinan, CHINA. \\ ${ }^{3}$ College of Pharmacy, South-Central University for Nationalities, Wuhan, 430074, CHINA. \\ Correspondence \\ Xu Ran}

School of biological and pharmaceutical engineering, Wuhan Polytechnic University, Wuhan City, 430023, CHINA.

Phone no: 86-15927308733

E-mail: xuran3006@126.com

\section{History}

- Submission Date: 26-04-2017;

- Review completed: 02-06-2017;

- Accepted Date: 22-08-2017

DOI : 10.5530/pj.2017.6.114

Article Available online

http://www.phcogj.com/v9/i6

\section{Copyright}

(C) 2017 Phcog.Net. This is an openaccess article distributed under the terms of the Creative Commons Attribution 4.0 International license.

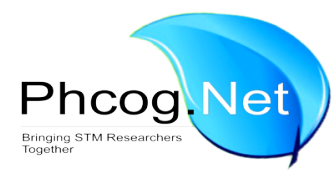

\begin{abstract}
To establish a method for quercetin content determination of the three plant drugs from genus Sedum and to decide their best harvesting time. Dried herbs of Sedum sarmentosum Bunge., S. lineare Thunb. and S. erythrostictum Migo. are all traditional medicines from Genus Sedum. They all have long been used to cure hepatitis, dysentery, swelling poison and so on. It has been reported that flavonoids contained in S. sarmentosum Bunge. were one type of the active components to protect liver and reduce serum alanine aminotransferase level. Through the qualitative analysis and TLC assay, we have verified that both $S$. lineare Thunb. and S. sarmentosum Bunge. contain flavonoid glycosides whose aglycones include quercetin. In this study, a HPLC methodology was established to determine quercetin which was a common hydrolyzate of the flalvonoid glycosides in the three plant medicines. The determination method developed showed good linearity in the range of $41.2-412.0 \mathrm{\mu g} / \mathrm{mL}$, and had a nice accuracy and repeatability. It indicated that the content of quercetine in Sedum sarmentosum, Sedum lineare and Sedum emarginatum can achieve the quality standard required by Chinese Pharmacopoeia.( No less than $0.01 \%$ ), The results also revealed preliminarily a relation between collected season and medicine quality. Thus it could be used to control some flavonoids content in the three plant drugs indirectly. By determining the samples of the 3 drugs collected in different seasons, the optimal harvest periods could be preliminarily ascertained.

Key words: Genus Sedum, Plant medicines, HPLC determination, Quercetin, Best harvest time.
\end{abstract}

\section{INTRODUCTION}

Sedum sarmentosum Bunge., S. lineare Thunb. and S. erythrostictum Migo., whose dried herbs are traditional herbal medicines, all belong to Genus Sedum. As the three plants are closely related with each other, they usually possess similar chemical components. Despite certain differences that existed among their efficacy, all could treat hepatitis, dysentery, swelling poison and so on. ${ }^{1-4}$ It has be proved that sarmentosin, a water-soluble component contained in S. sarmentosum Bunge., is one of the active components to protect liver and reduce serum alanine aminotransferase level. Many reports on the methods to determine sarmentosin have appeared, including to the IR, HPLC and GC approaches..$^{5-7}$ The studies also find that the flavonoids included in S. sarmentosum Bunge. ${ }^{8}$ show a better efficacy in liver protection and the enzyme decrease compared with the water-soluble total glycoside, ${ }^{9}$ suggesting that flavonoids should also be main active composition to treat hepatitis. Through the qualitative analysis and TLC assay, we have verified that both S. lineare Thunb. and S. erythrostictum Migo. also contained flavonoids. And the preliminary HPLC analysis has shown that the three plant drugs all contain the flavonoid glycosides whose aglycones include quer- cetin. As HPLC has been a routine methodology to determine the active composition in natural plants both at home and abroad, ${ }^{10-13}$ in this study, we have developed a HPLC means to determine the quercetin content of the samples collected in various seasons, trying to find out the variation trends of the some flavonoids content vs. the growing periods, obtain the appropriate harvest seasons and preliminarily control the quality of the 3 medicines finally.

\section{EXPERIMENTAL}

\section{Reagents and materials}

The quercetin reference substance was purchased from Chinese pharmaceutical and biological product verification station (batch number: 10008-200406); methanol and phosphoric acid employed here were HPLC grade reagents. Deionized (DI)water was used in the experiment.The three plant medicines collected from Jianshi, Huangmei, and Sheshan (Wuhan), Hubei province of China, and were respectively identified as the dried herbs of $S$. sarmentosum Bunge., S. lineare Thunb. and S. erythrostictum Migo. 
by Professor Wan Ding-rong from college of pharmacy, South-central University for Nationalities.(Table 1)

\section{Chromatographic conditions}

High performance liquid chromatography (HPLC) analysis was performed using an Agilent 1200 HPLC system from Agilent (Karlsruhe, Germany), equipped with a quaternary pump, an autosampler, and a VWD UV detector. Quercetin was separated from the sample solutions using a $\mathrm{C}_{18}$ column $(4.6 \times 250 \mathrm{~mm}$ I.D., particle size $5 \mu \mathrm{m}$, Agilent Eclips XDB- $\mathrm{C}_{18}$ ), with a mobile phase consisting of methanol and $0.40 \%$ phosphoric $\operatorname{acid}(49: 51, \mathrm{~V} / \mathrm{V})$, at $25^{\circ} \mathrm{C}$. The flow rate was $1.0 \mathrm{ml} / \mathrm{min}$, and injections were $20 \mu \mathrm{l}$ in volume.

\section{Standard solution preparation}

$10.3 \mathrm{mg}$ of the quercetin standard substance was accurately weighed into a $100 \mathrm{ml}$ volumetric flask and made up to the volume with methanol. After evenly mixed, $2 \mathrm{ml}$ was accurately transferred to a $10 \mathrm{ml}$ flask and was diluted with methenol to the volume. Thus, quercetin standard solution $(20.6 \mu \mathrm{g} / \mathrm{ml})$ was abtained.

\section{Sample preparation}

About $2.0 \mathrm{~g}$ of dried $\left(100^{\circ} \mathrm{C}\right.$, to a constant weight) of Sedum sarmentosum, S. lineare and S. erythrostictum. samples powder was taken and weighed accurately and respectively, then $20 \mathrm{ml}$ of methanol was added and refluxed twice( each time for $30 \mathrm{~min}$ ). The mixture was filtered into a $50 \mathrm{ml}$ volumetric flask. The residue was washed with methanol $(2 \times 10 \mathrm{ml})$, and transferred to the same flask, consecutively, and then made up to the volume with methanol, and shaken until evenly mixed. After that, $20 \mathrm{ml}$ was taken, mixed with $5 \mathrm{ml}$ of $25 \%$ hydrochloric acid solution, refluxed for $30 \mathrm{~min}$, then cooled immediately and transferred to a $50 \mathrm{ml}$ volumetric flask, and made up to the volume with methanol, finally shaken, filtered through a $0.45 \mu \mathrm{m}$ millipore filter prior to HPLC analysis.

\section{RESULTS}

Determination of the content of quercetin by HPLC

\section{Calibration curve}

A calibration curve was established for quercetin by injecting 2, 4, 8, $12,16,20 \mu \mathrm{l}$ of the standard solutions twice respectively. Linearity was tested by analyzing the average peak area of quercetin of different injection volume. And then the regression equation $(y=74.431 \mathrm{x}-0.9177)$ was obtained, and the correlation coefficient ( $r$ ) was 0.9999. Thus, a good linearity was shown when the quercetin concentration ranged from 41.2 to $412.0 \mu \mathrm{g} / \mathrm{ml}$. [Figure 1].

\section{Precision}

The precision was assessed by injecting $20 \mu \mathrm{l}$ of quercetin standard solution five times respectively. The quercetin content was calculated based on the calibration curve. The content variation (RSD, \%) was found to be $1.04 \%$, demonstrating that the instrument used had a high precision.

\section{Stability}

The $20 \mu \mathrm{l}$ of the same S. sarmentosum Bunge. sample (Jianshi, April) solutions were injected into the apparatus in a certain period of time $(0,2,4,6,8,12 \mathrm{~h})$ respectively, and quercetin content was calculated based on the calibration curve. As a result, the content variation (RSD, \%) was $1.65 \%$, revealing that the sample solution was stable within at least $12 \mathrm{~h}$.

\section{Repeatability}

Five same dried samples of the three plant medicines harvested in April were taken respectively to prepare the sample solutions according

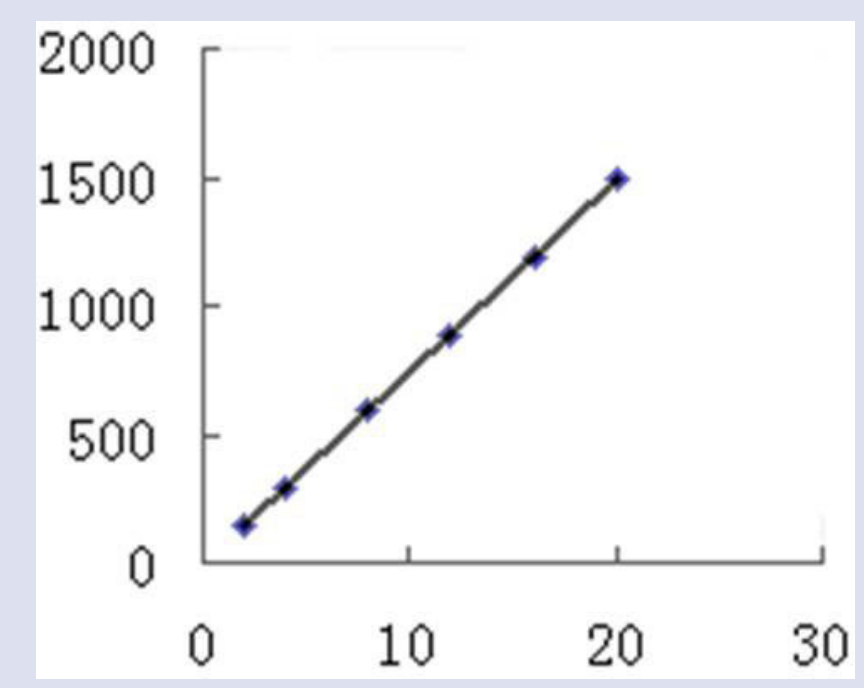

Figure 1: Calibration curve of standard quercetin

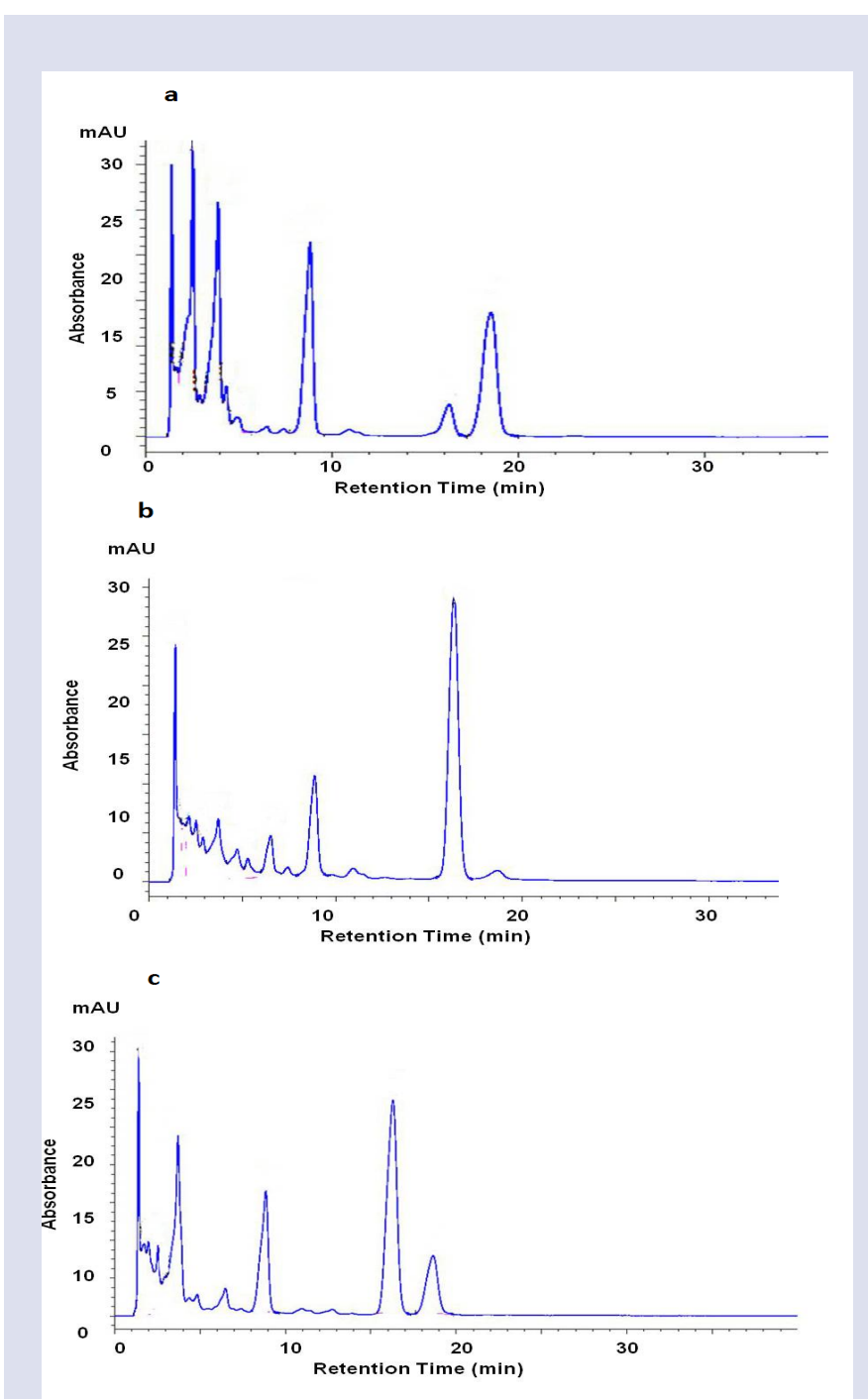

Figure 2: Typical HPLC chromatogram of S. sarmentosum collected in April (a: S. sarmentosum b: S. lineare c: S. erythrostictum). 


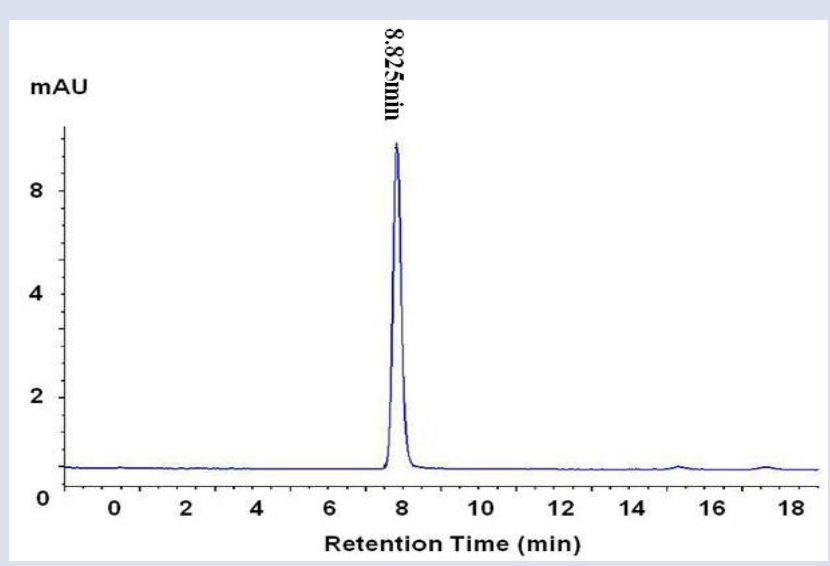

Figure 3: The quercetin standard substance HPLC peak.

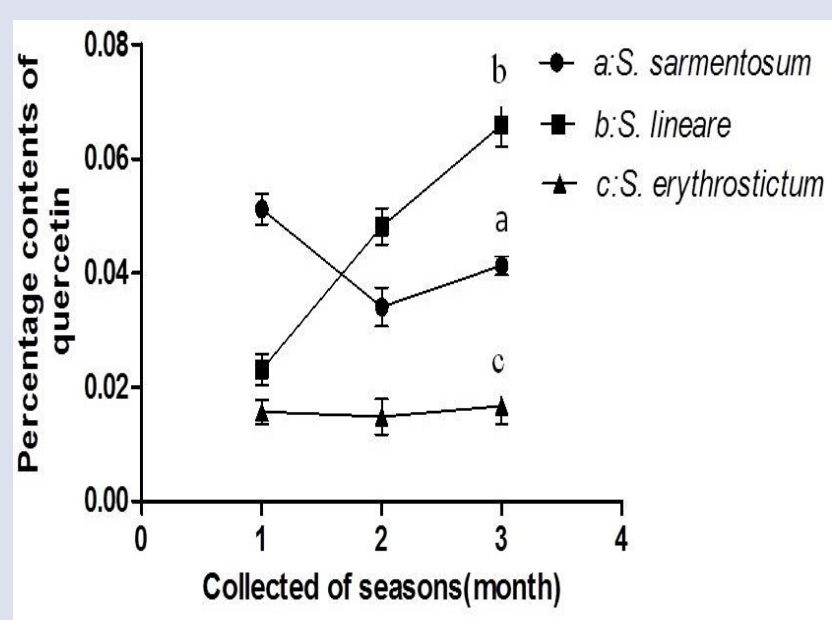

Figure 4: Percentage contents of quercetin contained in the 3 medicines collected in different seasons. (a: S. sarmentosum b: S. lineare c: $S$. erythrostictum)
Table 2: The quercetin content of the three herbal medicines

\begin{tabular}{ccccc}
\hline Species & $\begin{array}{c}\text { Collected } \\
\text { seasons }\end{array}$ & Habitat & Percentage & $\begin{array}{c}\text { Specimen } \\
\text { number }\end{array}$ \\
\hline S. sarmentosum & Apr. 27th & Huangmei, Hubei & $0.0512 \pm 0.003 \mathrm{a}$ & 120427 \\
S. sarmentosum & Jun. 28th & Sheshan, Wuhan & $0.0340 \pm 0.003 \mathrm{a}$ & 130628 \\
S. sarmentosum & Sep.15th & Sheshan, Wuhan & $0.0413 \pm 0.002 \mathrm{a}$ & 130915 \\
S. lineare & Apr. 2nd & Jianshi, Hubei & $0.0231 \pm 0.003 \mathrm{a}$ & 130402 \\
S. lineare & Jun. 29th & Sheshan, Wuhan & $0.0481 \pm 0.003 \mathrm{a}$ & 150729 \\
S. lineare & Oct. 5th & Sheshan, Wuhan & $0.0659 \pm 0.004 \mathrm{a}$ & 141005 \\
S. erythrostictum & Apr. 26th & Huangmei, Hubei & $0.0157 \pm 0.002 \mathrm{a}$ & 150426 \\
S. erythrostictum & Jun. 27th & Luotian, Hubei & $0.0148 \pm 0.003 \mathrm{~b}$ & 140627 \\
S. erythrostictum & Sep. 17th & Huangmei,Hubei & $0.0167 \pm 0.003 \mathrm{~b}$ & 150917 \\
\hline
\end{tabular}

${ }^{*}$ Different letters in same column were significantly different at $P<0.05$

to the method mentioned above prior to the HPLC determination. The consequences showed that the content variation RSD (\%) of each sample was less than $2.8 \%$, indicating a good repeatability of this method. [Figure 2, 3, 4]

\section{Recovery Test}

In the linear range, $1.0 \mathrm{mg}$ of quercetin which was nearly equal to that contained in $2.004 \mathrm{~g}$ of dried S. lineare Thunb. Sample collected in June in Huangmei was accurately taken to prepare the solution for recovery test according to the sample preparation method mentioned above. The quercetin content was determined according to the above chromatographic conditions. The recovery test was repeated six times. By comparing obtained quercetin content with that actually injected each time, the average recovery was $100.52 \%$, and RSD (\%) was $2.2 \%$, showing that developed determination method had a nice accuracy.

Average retain time of quercetin peak was $8.835 \mathrm{~min}$, and the quercetin standard substance HPLC peak was as follows [Figure 3].

\section{Sample analysis}

A total of 9 samples of three plant medicines collected in different periods were determined by the developed analytical method. The results of the quercetin content were given in the following table (Table 2). And the relations of the content change and the collected months were shown as follows [Figure 2].

\section{DISCUSSION}

All Sedum samples harvested in different seasons were weighed accurately, prepared according to the previously described method and analyzed by injecting thrice into the HPLC. The amounts of quercetin in these Sedum samples are listed in Table 1, and the variation with respect to the harvest season of the three Sedum medicinal plants is shown in Table 2. The results showed that the amounts of quercetin in Sedum sarmentosum, S. lineare and S. erythrostictum, and the content of quercetine in this three drugs can achieve the quality standard required by Chinese Pharmacopoeia.( No less than $0.01 \%$ ). In conclusion, the contents of quercetin varied in all Sedum medicinal samples harvested in different seasons. The content of quercetin was higher in Sedum sarmentosum harvested at the beginning of april, but lower in June, and grown a little bit at September, the highest content of quercetin reached 0.0512 percent at april, and then The content of quercetin in S. lineare and $S$. erythrostictum increased in the later harvest months, and the highest content of quercetin reached 0.0659 and 0.0167 percent at September respectively. 


\section{CONCLUSION}

By the HPLC analysis, we found that Sedum sarmentosum, S. lineare. and S. erythrostictum all contained flavonoid glycosides, whose hydrolysate included quercetin. The results suggested that the common flavonoids existed in the 3 plant medicines should be associated with anti-hepatitis activity. ${ }^{14,15}$

The results also revealed preliminarily a relation between collected season and medicine quality. In detail, the quercetin content of S. lineare Thunb. went up with growing months and reached the climax in October, while both S. erythrostictum Migo. and S. sarmentosum Bunge. had the relatively high amount in April (flowering period), and were the lowest in June (during this period, the growing state of S. sarmentosum Bunge. was poor, and the leaves mostly withered), then increased a little in September, showing preliminarily that the last two would own the best quality if collected in flowering period.

In a word, The HPLC assay of the quercetin in the hydrolysate of the 3 herbal drugs could be used to control the drug quality to a certain extent from the aspect of the related flavonoids content.

\section{ACKNOWLEDGMENT}

The author gratefully acknowledges the financial support by the Hubei Provincial Department of education plan for Science \& Technology Project (Grant no. Q20171705).

\section{CONFLICT OF INTEREST}

All contributing authors declare no conflicts of interest.

\section{ABBREVIATION USED}

TLC: Thin-layer chromatography; HPLC: High performance liquid chromatography; RSD: Relative standard deviation.

\section{REFERENCES}

1. Fang Z, Zhu S, Tan Z, etc. Selective Herbal from Enshi [M].Beijin: International Cultural Publishing Company. 2002;197-8.

2. Jia M, Li x. Ethnography to China[M]. Beijin: China Medical Science Press 2005,557

3. Fang $Z$, Zhao $H$, zhao J. Flora of Tujia nationality[M]. China Medical Science Press. 2007;748-9.

4. China Pharmacopeia (1977 edition, Volume I) [M].297

5. Liu S, Gao Q, Li J. "IR spectrum data software determination program usilization in pharmaceutical anlysis: IR spectrum determination of sarmentosin in Sedum sarmentosum Bunge." Journey of Pharmaceutical Analysis. 1984;4(5):276-8.

6. Lv X, Cao X, Zhang S, etc. "Analytical study on Sedum erythrostictum Migo. and its preparition". Pharmaceutical Journal. 1984;19(12):914-5.

7. Li J, Huang X, Ye C. "Determination of sarmentosin in Sedum erythrostictum Migo collected in different seasons and its related plants." Pharmaceutical Journal. 1984;16(5):268-70.

8. Aimin H, Wang M. "The flavonoids in Sedum erythrostictum Migo." Chinese Traditional and Herbal Drugs. 1997;28(9):517-22.

9. Yongheng $B$, Hong $L$, Liping $H$, etc. Effect of Sedum sarmentosum Bunge Extract on Aristolochic Acid-Induced Renal Tubular Epithelial Cell Injury". Journal of Pharmacological Sciences. 2014;124(445-456):365-6.

10. Dongmei W, Fengyuan He, Zhenjiang Lv, etc. "Phytochemical Composition, Antioxidant Activity and HPLC Fingerprinting Profiles of Three Pyrola Species from Different Regions." Plos One. 2014;9(5):1-12.

11. Tung-Y, Fang-R, Jing-R. "Rapid HPLC Quantification Approach for Detection of Active Constituentsin Modern Combinatorial Formula,San-Huang-Xie-XinTang(SHXXT)". Frontiers in Pharmacology. 2002;35(15):2459-70.

12. Andrzej L. Dawidowicz, Dorota W, Jan G, Danuta HS. “Optimization of ASE conditions for HPLC of rutin and isoquercitrin in Sambucus nigra L... Journal of Liquid Chromatography \& Related Technologies. 2016;7(20):374-90.

13. Yin-Ping W, Xian-SM, Yong-Rui B, Shuai W, Ting-GK. Simultaneous Quantitative Determinationof Nine Active Chemical Compositions in Traditional Chinese Medicine Glycyrrhiza by RP-HPLC with Full-Time Five-Wavelength Fusion Method. The American Journal of Chinese Medicine. 2002;41(1):1211-9.

14. Hubei food and drug administration. Quality standards for traditional Chinese herbal medicine of Hubei province (2009 edition), Hubei Science and technology press, 2009, 33

15. China Pharmacopeia ( 2015 edition, Volume I). 148

\section{GRAPHICAL ABSTRACT}

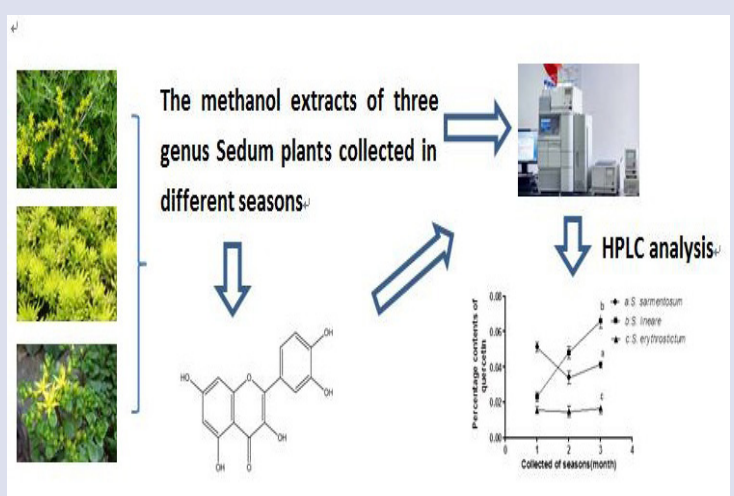

\section{SUMMARY}

- To establish a method for quercetin content determination of the three plant drugs from genus Sedum and to decide their bestharvesting time. It indicated that the content of quercetine in Sedum sarmentosum, Sedum lineare and Sedum emarginatum can achieve the quality standard required by Chinese Pharmacopoeia. The results also revealed preliminarily a relation between collected season and medicine quality. Thus it could be used to control some flavonoids content in the three plant drugs indirectly.

- The HPLC assay of the quercetin in the hydrolysate of the 3 herbal drugs could be used to control the drug quality to a certain extent from the aspect of the related flavonoids content.

\section{ABOUT AUTHORS}

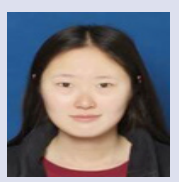

Yue-ling Ma: Presently working as a undergraduate student in the School of biological and pharmaceutical engineering, Wuhan Polytechnic University, Wuhan, China. Her area of expertise and interest includes identification and quality evaluation of traditional chinese medicine, research and development of new biologically active substances.

Cite this article: Yue-ling M, Yu-jie C, Ding-rong W, Ping C, Ran X. HPLC determination of quercetin in three plant drugs from genus Sedum and conjecture of the best harvest time. Pharmacog J. 2017;9(6):725-8. 